\title{
Immuno Nanoparticles Integrated Electrical Control of Targeted Cancer Cell Development Using Whole Cell Bioelectronic Device
}

\author{
Evangelia Hondroulis' ${ }^{1}$, Rui Zhang1, Chengxiao Zhang'ㄹ, Chunying Chen ${ }^{3}$, Kosuke Ino ${ }^{4}$, Tomokazu \\ Matsue $^{4,5}$, Chen-Zhong $\mathrm{Li}^{1,2,5} \bowtie$ \\ 1. Nanobioengineering/Bioelectronics Laboratory, Department of Biomedical Engineering, Florida International University, 10555 West \\ Flagler Street, Miami, FL 33174, USA \\ 2. Key Laboratory of Applied Surface and Colloid Chemistry, Ministry of Education, School of Chemistry and Chemical Engineering, \\ Shaanxi Normal University, Xi'an 710062, China \\ 3. CAS Key Lab for Biomedical Effects of Nanomaterials and Nanosafety, National Center for Nanoscience \& Technology of China, Beijing \\ 100190, PR China \\ 4. Graduate School of Environmental Studies, Tohoku University \\ 5. WPI Advanced Institute for Materials Research, Tohoku University, Sendai 980-8577, Japan
}

$\bowtie$ Corresponding author: E-mail: licz@fiu.edu, 305-348-0120

(ㅇ Ivyspring International Publisher. This is an open-access article distributed under the terms of the Creative Commons License (http://creativecommons.org/ licenses/by-nc-nd/3.0/). Reproduction is permitted for personal, noncommercial use, provided that the article is in whole, unmodified, and properly cited.

Received: 2014.01.13; Accepted: 2014.06.04; Published: 2014.07.13

\begin{abstract}
Electrical properties of cells determine most of the cellular functions, particularly ones which occur in the cell's membrane. Manipulation of these electrical properties may provide a powerful electrotherapy option for the treatment of cancer as cancerous cells have been shown to be more electronegative than normal proliferating cells. Previously, we used an electrical impedance sensing system (EIS) to explore the responses of cancerous SKOV3 cells and normal HUVEC cells to low intensity $(<2 \mathrm{~V} / \mathrm{cm})$ AC electric fields, determining that the optimal frequency for SKOV3 proliferation arrest was $200 \mathrm{kHz}$, without harming the non-cancerous HUVECs. In this study, to determine if these effects are cell type dependant, human breast adenocarcinoma cells (MCF7) were subjected to a range of frequencies $(50 \mathrm{kHz}-2 \mathrm{MHz})$ similar to the previously tested SKOV3. For the MCF7, an optimal frequency of $100 \mathrm{kHz}$ was determined using the EIS, indicating a higher sensitivity towards the applied field. Further experiments specifically targeting the two types of cancer cells using HER2 antibody functionalized gold nanoparticles (HER2-AuNPs) were performed to determine if enhanced electric field strength can be induced via the application of nanoparticles, consequently leading to the killing of the cancerous cells without affecting non cancerous HUVECs and MCFIOa providing a platform for the development of a non-invasive cancer treatment without any harmful side effects. The EIS was used to monitor the real-time consequences on cellular viability and a noticeable decrease in the growth profile of the MCF7 was observed with the application of the HER2-AuNPs and the electric fields indicating specific inhibitory effects on dividing cells in culture. To further understand the effects of the externally applied field to the cells, an Annexin V/EthD-III assay was performed to determine the cell death mechanism indicating apoptosis. The zeta potential of the SKOV3 and the MCF7 before and after incorporation of the HER2-AuNPs was also obtained indicating a decrease in zeta potential with the incorporation of the nanoparticles. The outcome of this research will improve our fundamental understanding of the behavior of cancer cells and define optimal parameters of electrotherapy for clinical and drug delivery applications.
\end{abstract}

Key words: biosensor, electrotherapy, cancer, antibody, nanoparticles 


\section{Introduction}

The effects of exogenous electric fields on physiology and their possible relationship to diseases have interested researchers for years [1-3]. In 1855, Guillaume Duchenne discovered that alternating current leads to electrotherapeutic triggering of muscle contractions thus spurring the use of electrical energy as medical treatment. Since then, the use of electric fields has become popular in fields such as gene and cellular therapies [4-6], and has even progressed to clinical trials for drug delivery [7], however; still little is known how electric fields may interact with intracellular signaling pathways to potentially alter cell physiology.

The idea of classifying cancers by their electrical properties was first proposed by Fricke and Morse in 1926 [8]. Recently, there has been interest in the use of electrotherapy as a non-surgical and minimally invasive treatment for cancer, since the electrical and physical properties of cancer cells differ from normal proliferating cells. Electrical properties of cells determine most of the cellular functions, predominantly proliferation and differentiation. In particular, the transmembrane potential, the voltage difference across the membrane produced by the balance of intracellular and extracellular ionic concentrations, is responsible for controlling mitosis, DNA synthesis, and the majority of other cell cycle phenomena [9]. Several studies have previously been done to derive the relationship between transmembrane potential and cell proliferation. Initially, Cone and Tongier (1973) investigated the effects of transmembrane modification on the mitotic activity of Chinese hamster ovary cells [10]. Their study showed that decreasing the cells transmembrane potential ultimately stopped the mitotic process in the cells with the process being reversible once the transmembrane potential returned to a normal value. More recently, MCF-7, human adenocarcinoma, membranes have been shown to hyperpolarize during the G0/G1 phase of proliferation [11]. Manipulation of these electrical properties may provide a powerful electrotherapy option for the treatment of cancer as cancerous cells have been shown to have more electronegative membrane potential than normal proliferating cells, thus, further studies on cancer electrotherapy is warranted.

Current treatments for cancer have much potential; however, a major limitation in these treatments is the negative side effects that occur. Electrotherapy for cancer treatment is very promising alternative as it eliminates the toxic chemicals and possible immunogenic responses in the host tissue. Current research for cancer electrotherapy mostly focuses on using short electric pulses to alter cell physiology, in particular, the permeabilization of the cell membrane. This phenomenon is then modified either by the addition of chemotherapy agents [12], by inducing apoptosis of the cells [13] or even for DNA vaccination against certain cancer types [14-15]. However, there are limitations with this technique as it can only be efficient if all tumor cells are permeabilized, and thus there is also a size dependence of the tumor as smaller tumors show a higher response rate to the therapy [16]. Also, the electric field has to be as low as possible to ensure the safety of the procedure to prevent unwanted side effects such as erythema, edema, superficial epidermal erosion, or scars [12].

Previously, an electrical impedance sensor (EIS) was used to explore the responses of cancerous SKOV3 cells and normal HUVEC cells to low intensity $(<2 \mathrm{~V} / \mathrm{cm})$ AC electric fields, determining that the optimal frequency for SKOV3 proliferation arrest was $200 \mathrm{kHz}$, without harming the non-cancerous HUVECs [17]. A slower proliferation rate was observed in the cancer cells through the lower resistance curves of the EIS in real-time as the external field was applied compared to a control with no applied field (Scheme 1 /Figure 8). In this study, we determined if the effects of the externally applied electric field are cell type dependant using human breast adenocarcinoma cells (MCF7). The MCF7, along with non cancerous MCF10a, were subjected to the range of frequencies $(50 \mathrm{kHz}-2 \mathrm{MHz})$ similar to the previously tested SKOV3 and HUVEC to determine the optimal frequency that would most hinder the proliferation of the MCF7. Monitoring the behavior of different types of cancerous cells under the effects of the electric field can lead to specific treatment paradigms for various types of cancers.

The ultimate fate of these cells after they endure the effects of the applied field is the next focus of this study. Nanomaterials are being considered in the development of new drugs and new therapies for disease control and improving the quality of life [18-20]. More recently, nanomaterials have been used in tissue engineering and medical imaging, leading to improved diagnostics and new therapeutic treatments [21-23]. Nanoparticles have been extensively studied for use in biomedical applications, particularly gold nanoparticles (AuNPs), due to their low cytotoxicity [24], specifically for use in antibody targeted drug delivery to cells [25]. For example, HER2, human epidermal growth factor receptor 2 , is a cell membrane surface-bound receptor tyrosine kinase that is responsible for intracellular signal transduction and ultimately cell growth and differentiation [26]. HER2 over-expression leads to cancerous cell proliferation and is seen in cancers such as breast cancer, ovarian cancer, stomach cancer, and even lung cancer [27], 
and both SKOV3 and MCF7 cells over-express HER2 markers on their surface membrane. It has also been shown that in the presence of an electric field, AuNPs could change the distribution of the field within the cell's cytoplasm resulting in an inward net electric force which potentially could change the function of the cell [28]. Thus, further experiments specifically targeting the two types of cancer cells using HER2 antibody functionalized gold nanoparticles (HER2-AuNPs) were performed to determine if enhanced electric field strength can be induced via the application of nanoparticles, consequently leading to the killing of the cancerous cells without affecting non cancerous HUVECs and MCF10a providing a platform for the development of a non-invasive cancer treatment without any harmful side effects.

The EIS was used to monitor the real-time consequences on cellular viability under the electric field with the incorporation of the HER2-AuNPs. The binding of charged nanoparticles to the cell surface plasma membrane will change the zeta potential value of the cells, a feature of the cell that has been used in cell biology to study cell adhesion, activation, and agglutination based on cell-surface-charge properties [29]. Thus the zeta potential of the AuNPs, HER2-AuNPs and each cell type before and after incorporation of the HER2-AuNPs was taken to monitor this phenomenon. The HER2-AuNPs would bind to the HER2 receptors on the membrane of the cancer cells, thus affecting the cells' zeta potential potentially producing a larger effect of cell viability of the cancerous cells with the application of the electric field to the combination of HER2-AuNPs and cell. The cell death mechanism was also tested by using an Annexin V/EthD-III assay to determine if the cells undergo apoptosis or necrosis after the application of the applied electric field.

\section{Experimental Details}

\section{Cell culture}

According to the American Cancer Society, every year over 200,000 American women are diagnosed with breast cancer, thus MCF7, human breast adenocarcinoma were chosen as another type of cancer cell for comparison with the SKOV3. HTB-77TM (SKOV3) and CRL-1730 ${ }^{\mathrm{TM}}$ (HUVEC) cells were purchased from American Type Culture Collection (ATCC, Rockville, MD, USA) and were cultured in McCoy's 5A Modified Medium and Dulbecco's Modified Eagle Medium respectively, each containing $10 \%$ fetal bovine serum, $0.3 \mathrm{mg} \mathrm{ml}^{-1} \mathrm{~L}$-glutamine, $100 \mathrm{U} \mathrm{ml}^{-1}$ penicillin and 100 $\mathrm{mg} \mathrm{ml}^{-1}$ streptomycin. The HTB-22 ${ }^{\mathrm{TM}}$ (MCF7) and CRL-10317TM (MCF10a) were graciously donated from the Research Institute at Miami Children's Hos- pital, Miami, FL, and were both cultured in Eagle's Minimum Essential Medium containing $10 \%$ fetal bovine serum, $0.3 \mathrm{mg} \mathrm{ml}^{-1} \mathrm{~L}$-glutamine, $100 \mathrm{U} \mathrm{ml}^{-1}$ penicillin and $100 \mathrm{mg} \mathrm{ml}^{-1}$ streptomycin. The cell cultures were placed in an incubator $\left(37^{\circ} \mathrm{C}, 5 \% \mathrm{CO}_{2}\right.$ atmosphere) for 24 hours prior to the experiment so that the cells reached confluency with a final concentration of $10^{5}$ cells ml-1. $0.4 \mathrm{ml}$ of cell suspension was inoculated into each well in the EIS chip for the experiments.

\section{Electrical impedance sensing (EIS)}

The EIS chip design was previously reported [25]. In short, individual tissue culture wells of volume $9 \times 9 \times 10 \mathrm{~mm}^{3}$ are placed over an array of eight detecting gold electrodes ( $250 \mu \mathrm{m}$ in diameter) each linked to a gold counter electrode $\left(7 \times 46 \mathrm{~mm}^{2}\right)$.

\section{Application of external electric field}

The design of the application of the external electric field to the EIS chip was previously described [17]. In brief, a unique system of a pair of insulated wires (BLK KYNAR 100', conductor area $0.25 \mathrm{~mm}$ ) was designed to be placed in conjunction with the 8 well array design of the EIS chip. The wires were placed in 4 of the wells with the other 4 wells used as the control. The wires were placed $1 \mathrm{~mm}$ apart to allow spacing to encompass the whole electrode area. A Leader LFG-1300S Function Generator was used to provide the square $\mathrm{AC}$ waves at the desired frequencies.

\section{Synthesis of AuNPs}

Hydrogen tetrachloroaurate $\left(\mathrm{HAuCl}_{4}\right)(40 \mathrm{ml}, 1.0$ $\mathrm{mM})$ was added to an Erlenmeyer flask $(250 \mathrm{ml})$, stirred and brought to the boil on a hotplate. Trisodium citrate $\left(\mathrm{Na}_{3} \mathrm{C}_{6} \mathrm{H}_{5} \mathrm{O}_{7}\right)(4 \mathrm{ml}, 1 \%)$ was added to the boiling solution. Three minutes after the addition of trisodium citrate, and 10 minutes of stirring, AuNPs were formed. The size of the AuNPs was characterized by the Zetasizer (Malvern Instruments, Woodstock, GA) and TEM and found to be $20 \mathrm{~nm}$, the size indicated in the HER2 functionalization procedure.

\section{HER2 antibody functionalization of AuNPs}

The HER2 antibody preparation was done following the procedure of $\mathrm{Zhu}$ et al. [30]. In short, The AuNPs solution was concentrated $5 \mathrm{X}$ and the $\mathrm{pH}$ of the AuNPs solution for antibody labeling was adjusted to pH $8.5 \sim 9.0$ with $0.2 \mathrm{ml}$ of $\mathrm{K}_{2} \mathrm{CO}_{3}(0.1 \mathrm{M})$. Purified Anti-ErbB2/Her2 Monoclonal Antibody (5 $\left.\mu \mathrm{l}, 1 \mathrm{mg} \mathrm{ml}^{-1}\right)$ was added to the AuNPs solution (750 $\mu \mathrm{l}, 5 \mathrm{X}$ ) and stirred gently at room temperature for $1 \mathrm{~h}$. The conjugate was stabilized by adding BSA ( $90 \mu \mathrm{l}$, $10 \%)$ in sodium borate $(20 \mathrm{mM})$ for a final concentration of $1 \%$ and the solution was incubated for another 
$15 \mathrm{~min}$. The mixture was then centrifuged at $7000 \mathrm{rcf}$ for $15 \mathrm{~min}$. Two phases can be obtained: a clear to pink supernatant of unbound antibodies and a dark red, loosely packed sediment of the AuNPs-Antibody conjugates. The supernatant was discarded and the pellet was resuspended in BSA/PBS $(100 \mu \mathrm{l}, 1 \%)$. After another centrifugation at $7000 \mathrm{rcf}$ for $15 \mathrm{~min}$, the supernatant was removed and the pellet was resuspended in $100 \mu \mathrm{l}$ buffer, containing sodium phosphate $(20 \mathrm{mM})$, Tween $20(0.25 \%)$, sucrose $(10 \%)$, and BSA (5\%). The conjugate was stored at $4^{\circ} \mathrm{C}$ until required for use.

\section{Apoptosis / Necrosis Assay}

Apoptotic/Necrotic Cells Detection Kit was purchased from PromoKine (Germany). The kit contains FITC-Annexin $\mathrm{V}$ in TE buffer containing $0.1 \%$ BSA and $0.1 \% \mathrm{NaN}_{3}$ (pH 7.5), EthidiumHomodimer III (EthD-III) in PBS and 5X Binding Buffer. A 1:5 dilution of Binding Buffer to DI water was made. After runs with the EIS, the cells were detached from the wells on the chip with $0.25 \%$ Trypsin/EDTA and suspended with the 1:5 Binding Buffer/DI solution giving a final concentration of $10^{5}$ cells ml-1. $100 \mu \mathrm{l}$ of the cell suspension was placed in a microcentrifuge tube to which $5 \mu \mathrm{l}$ of FITC-Annexin V and $5 \mu \mathrm{l}$ of EthidiumHomodimer III solutions were added to each tube. The samples were incubated at room temperature for 15 minutes in the dark, after which the cells were washed and resuspended with the 1:5 Binding Buffer/DI solution. Fluorescence intensity was determined using a confocal microscope (Perkin Elmer UltraViewVox system, USA) with FITC and Texas Red filter sets.

\section{Zeta potential measurement}

The ZetasizerNano ZEN 3600 (Malvern Instruments, United Kingdom) was used to measure the zeta potential of the AuNPs, HER2-AuNPs and the HER2-AuNPs with each cell type, SKOV3, HUVEC, MCF7 and MCF10a.

\section{AFM imaging and analysis}

AFM images of live cells were obtained in fluid tapping mode using a Nanoscope IIIa multimode system (Veeco Metrology, CA). The cells were grown to confluency on Poly-L-Lysine coated glass coverslips. The AFM cantilever was positioned on four different regions of the cell surface to acquire $5 \mu \mathrm{m} \times 5$ $\mu \mathrm{m}$ topographical and amplitude images. Qualitative analysis of the particles was performed by visualization of the amplitude and topographical images and by quantitative analysis of roughness measurements from the topographical images. Roughness values of the cell surface were calculated from the topographical image in an offline mode using Nanoscope Image
Analysis software (Version 5.13r1; Veeco Metrology). Average cell membrane surface roughness measurements were obtained at various time points representing characteristics of the cell surface influenced by $\mathrm{NP}$ attachment and internalization. A series of topographical images of SKOV3 cell surfaces were obtained when the cells were incubated in the medium with and without AuNPs.

\section{Results}

\section{Optimal frequency for MCF7}

As done before with the SKOV3 cells, the MCF7 and MCF10 cells were subjected to five different frequencies of alternating electric fields 50, 100, 150 and $200 \mathrm{kHz}$ and $2 \mathrm{MHz}$, to find the optimal frequency that would most hinder the growth of the MCF7 cells without harming the normal MCF10a cells. Figure 1 shows the EIS results of the MCF10a (1 (a), (c)) and the MCF7 (1 (b), (d)) for the intermediate frequencies (100, $150,200)$ and the extreme frequencies $(50 \mathrm{kHz}, 2 \mathrm{MHz})$ respectively.

The breast cancer cell lines behaved in a similar manner to the ovarian cancer cell lines tested previously as did the noncancerous MCF10a to the HUVECs. Under the influence of the electric field at all five frequencies, the resistance values for the MCF10a cells followed a trend of proliferating cells gradually attaching and spreading across the electrode surface creating an increase in resistance values. This can be seen in Figures 1 (a) and (c) which show the resistance values of the MCF10a when exposed to the intermediate range frequencies $(100,150,200 \mathrm{kHz})$ and the extreme frequencies $(50 \mathrm{kHz}, 2 \mathrm{MHz})$ respectively. However, the MCF7 exhibited a variation of trends in resistance profiles with the different frequencies of the applied field. Shown in Figure 1 (b), 150 and $200 \mathrm{kHz}$ decreased the resistance profiles of the MCF7, however, the MCF7 have the lowest resistance profile with $100 \mathrm{kHz}$ indicating the most affect on the cell proliferation at this frequency. The extreme frequencies were also tested to justify that the intermediate frequencies are the target frequencies suitable for cancer electrotherapy. The MCF10a cells retained a similar profile with the applied field as without (Figure 1 (c)). In Figure $1(\mathrm{~d})$, the MCF7 show more of a difference when the extreme frequencies are applied compared to the previously tested SKOV3. The higher frequency of $2 \mathrm{MHz}$ had an effect on the MCF7 proliferation as can be seen in the lower resistance profile compared to the control with no field applied. This could be due to the more sensitive membrane of the MCF7 compared to that of the other types of cancer cells. Cancer cells, in general, have lower membrane potentials with lower stiffness compared to their normal coun- 
terparts. This is seen in the Young's modulus of each type of membrane using atomic force microscopy (AFM) [31]. In addition, the MCF7 breast cancer cells in general, were observed to have a lower Young's modulus in comparison with other types of cancer cells, potentially giving the cells a weaker membrane. $50 \mathrm{kHz}$ also slowed down the proliferation of the MCF7 cells; however, the $100 \mathrm{kHz}$ affected the proliferation of these cells the most.

Digital images of the cells were also taken for the MCF10a and MCF7 cells with and without exposure of the $100 \mathrm{kHz}$ applied field. This was done to get a visual understanding of the cell behavior on the EIS electrode surface as the electric field is applied. The changes in resistance profiles of the cells with and without the applied field could be due to a slower proliferation rate caused by the exposure to the field, or simply, by the detachment of the cells from the electrode. Figure 2 shows one well each of the MCF10a cells after 20 hours without the applied field (a), after 20 hours with the applied $100 \mathrm{kHz}$ field (c), the MCF7 cells after 20 hours without the applied field (b), and after 20 hours with the applied $100 \mathrm{kHz}$ field (d). We see the MCF10a maintain a solid monolayer over the electrode after 20 hours of field expo-

(a)

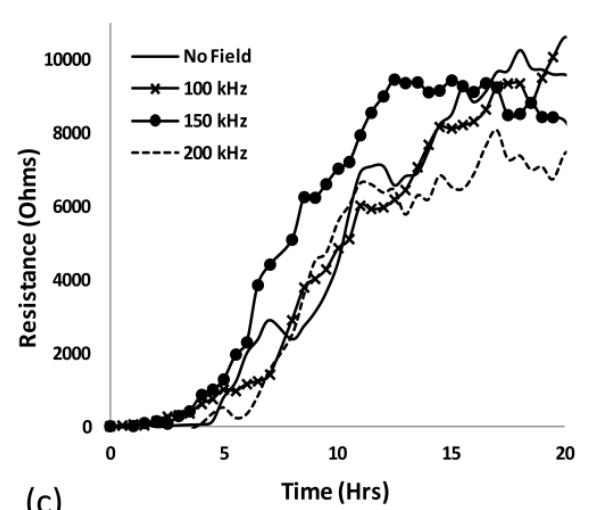

(c)

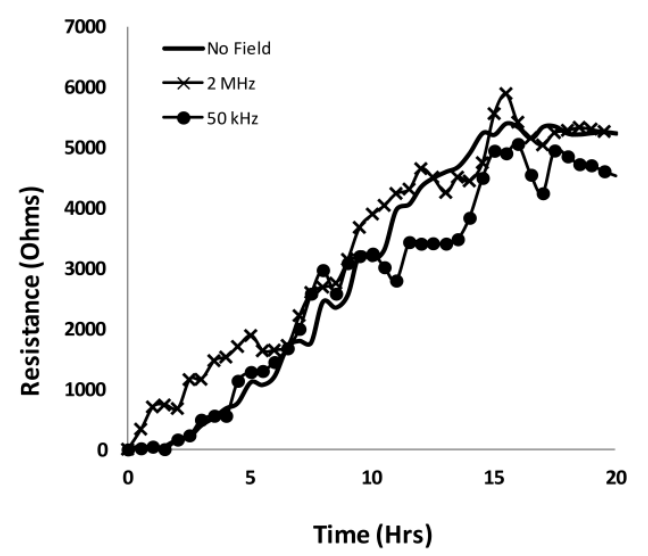

sure. However, for the MCF7, we do not see a solid monolayer attached over the electrode. In the case of cell detachment, there would be evidence of the detached cells floating in the surrounding media, thus indicating the externally applied field having a detrimental affect towards the cell proliferation.

\section{HER2-AuNPs targeted enhancement}

Gold nanoparticles are being use in targeted therapies in biomedical engineering such as gene and drug delivery transfection vectors, DNA-binding agents and in various imaging systems due to their inert properties [32]. In this study, we functionalized our AuNPs with a HER2 antibody for a targeted delivery of the particles to the cell membrane since HER2 is overexpressed in both breast and ovarian cancers, and based on the length of the experiment, the AuNPs were assumed to remain on the membrane surface during this experiment. We tested the two types of cancer cells, SKOV3 and MCF7, as well as the non cancerous HUVECs and MCF10a, under their respective optimal frequencies, 200 and $100 \mathrm{kHz}$, with and without the integration if the HER2-AuNPs to determine any enhancement in the effects of the applied electric field.

(b)
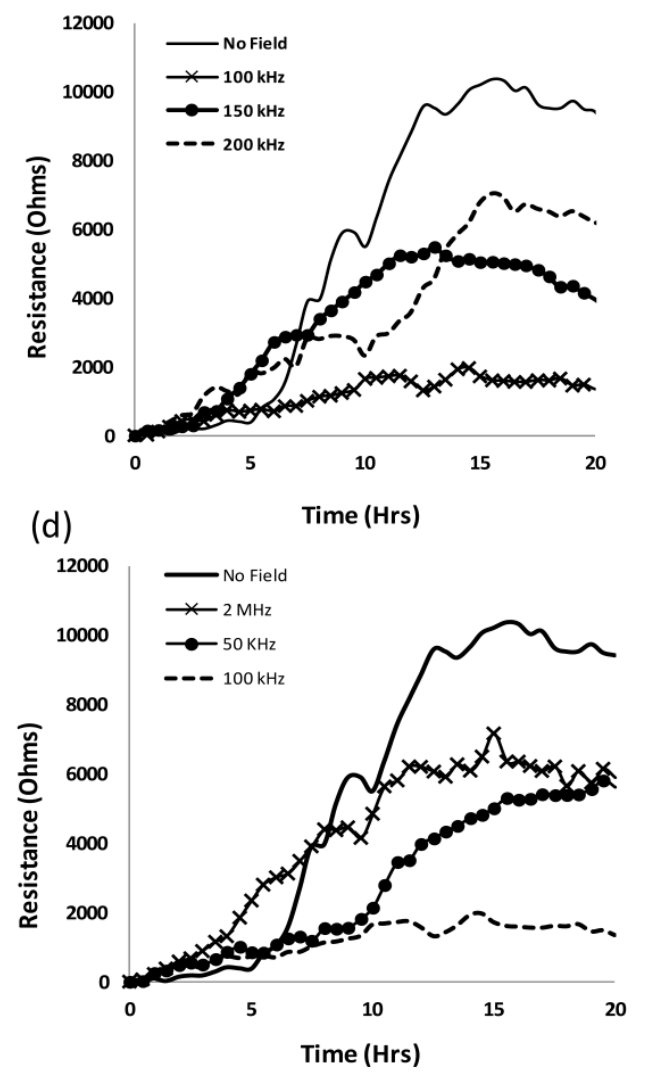

Figure I. Resistance values of MCFIOa (a) and MCF7 (b) under electric fields of frequencies 100 , 150 and $200 \mathrm{kHz}$. No apparent effect was noticed for the normal MCFIOa, however, a significant decrease in resistance values was observed for the MCF7 for all three frequencies. $50 \mathrm{kHz}$ and $2 \mathrm{MHz}$ frequencies were tested on both MCFIOa (c) and MCF7 (d). The MCFIOa showed no apparent effect on the cell proliferation as seen in the similar resistance curves to the control. I00 kHz showed the largest decrease out of all the frequencies for the MCF7. 


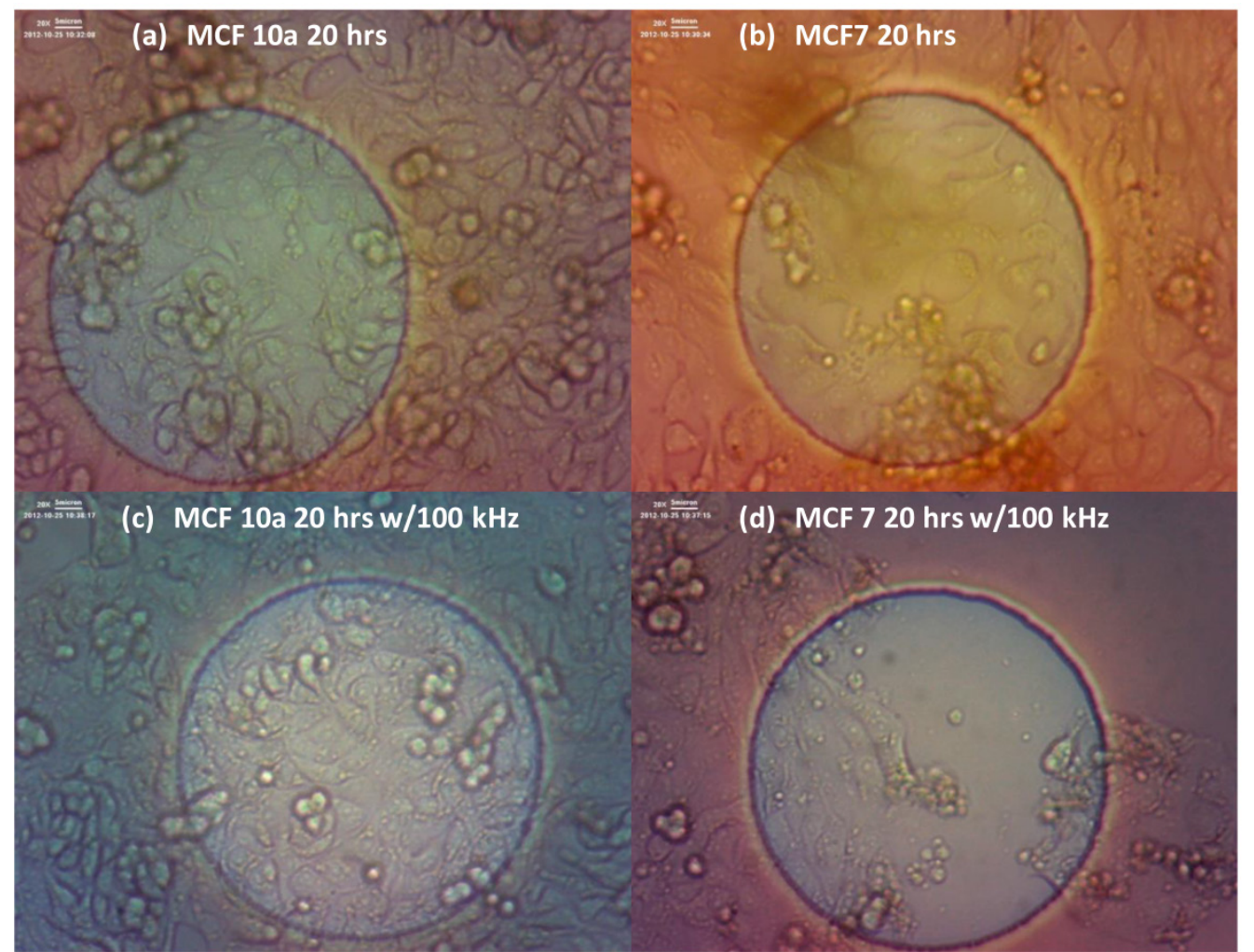

Figure 2. MCF IOa cells after 20 hours without the applied field (a), after 20 hours with the applied $100 \mathrm{kHz}$ field (c), the MCF 7 cells after 20 hours without the applied field (b), and after 20 hours with the applied $100 \mathrm{kHz}$ field (d). We see the MCF I0a maintain a solid monolayer over the electrode after 20 hrs of field exposure. However, for the MCF 7, we do not see a solid monolayer attached over the electrode indicating a decrease in cell proliferation. In the case of cell detachment, there would be evidence of the detached cells floating in the surrounding media.

The first concern with using the metallic nanoparticles under the applied electric field would be the potential heating of the nanoparticles. AuNPs are being used for cancer hyperthermia studies [33], however, these studies use nearinfrared (NIR) laser light, [34] radiowaves, [35] or even ultrasound [36]. To make sure temperature would not be a factor for the cancer cells under the intermediate frequencies determined for the two types of cancer cells, we tested the temperature of the wells at incremental times of 6 hours for the SKOV3 and 4 hours for the MCF7 for an entire run. Figure 3 (a) and (b) shows the average temperature of the 8 wells on one chip for the SKOV3 and the MCF7, respectively. Here we do not see any significant change in the tested temperatures when the HER2-AuNPs were incorporated for either type of cell, indicating no apparent thermal effects from the nanoparticles. Figure 4 (a) and (c) shows the resistance values obtained for the noncancerous HUVECs and MCF10a, respectively. As in the previous experiment, there is no noticeable change in the resistance values when the electric field was applied to the cells compared to the control cells with now applied field. Also, with the incorporation of the HER2-AuNPs, there is still no apparent effect in the resistance profiles. However, a noticeable decrease in the growth profiles of the SKOV3 and MCF7 was observed with the application of the HER2-AuNPs and the electric fields compared to just the field itself indicating specific inhibitory effects on dividing cells in culture (Figure 4 (b) and (d)).

Gold nanoparticles with sizes ranging from a few nanometers up to one hundred nanometers are most commonly used for study cell-nanoparticle interactions. To achieve effective targeting, the HER2 antibody functionalized AuNPs with a series of sizes are tested. Transmission electron microscopy (TEM) analysis, AFM, and the Zetasizer (Malvern Instruments, Woodstock, GA) were performed for the determination of particle sizes. Over 100 particles were counted in multiple pictures from different areas of the TEM grid resulting a size variation of $\pm 10 \%$. The detail of nanoparticle preparation and characterization can be found in our previous publication (17, 46-48). We found that the antibody functionalized AuNPs in the size range from 10 to $30 \mathrm{~nm}$ covered cell surface more uniformly and efficiently than the particles outside this range. Instead of casting cell surface, particles with size bigger than $30 \mathrm{~nm}$ are found to be more easily entering cells via endocytosis process leading to less effect on cells upon electrical field stimulation. 
(a)

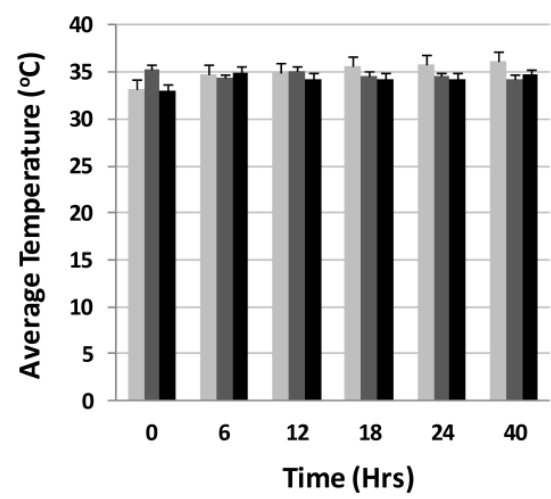

(b)

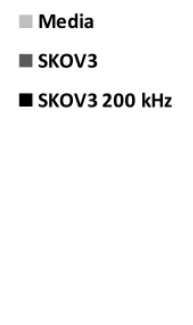

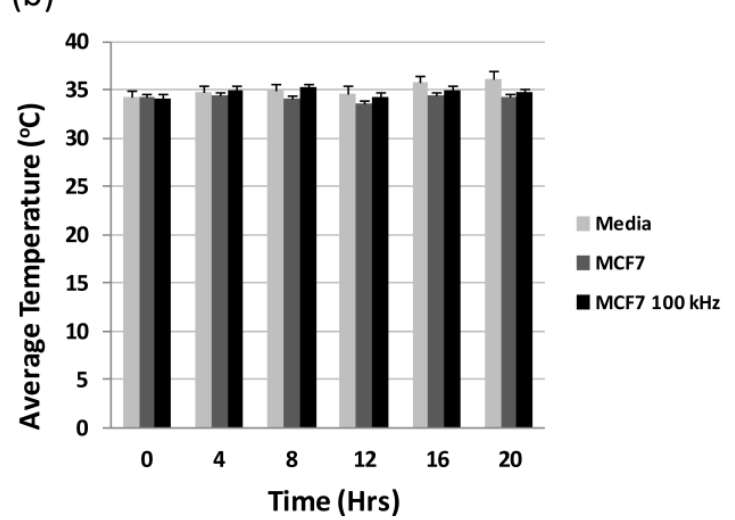

Figure 3. Average temperature readings for the 8 wells on a chip at various time points throughout an EIS run for SKOV3 (a) and MCF7 (b).

(a)

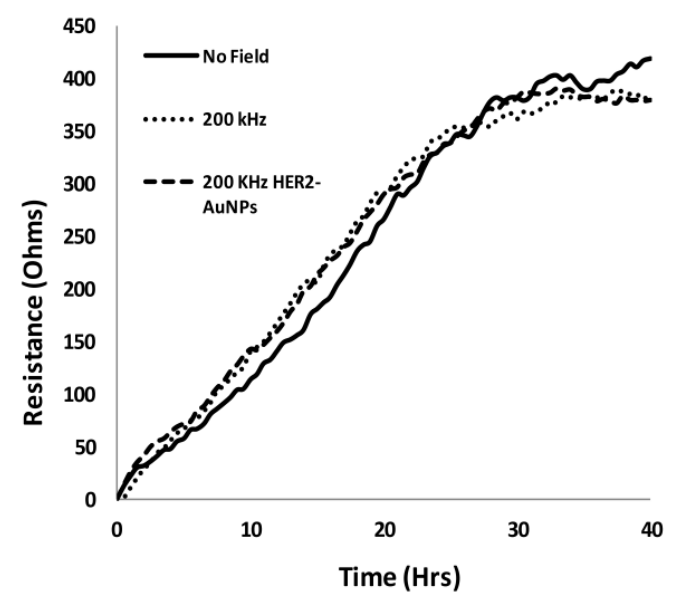

(c)

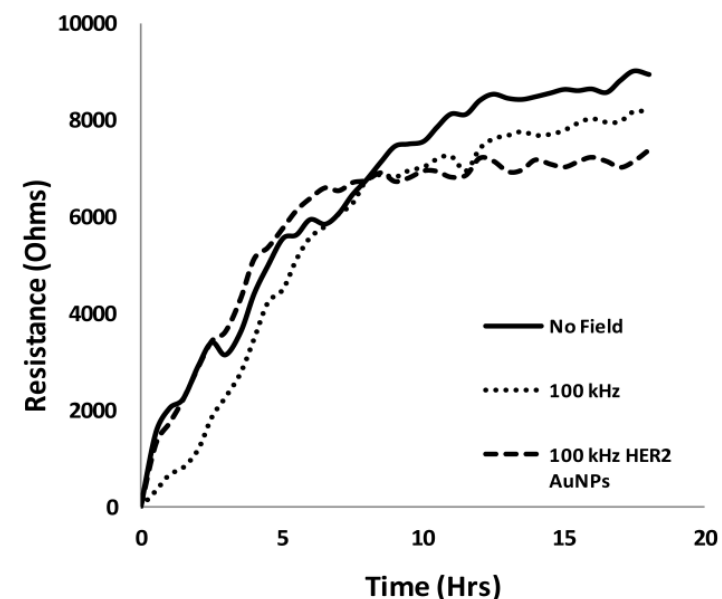

(b)

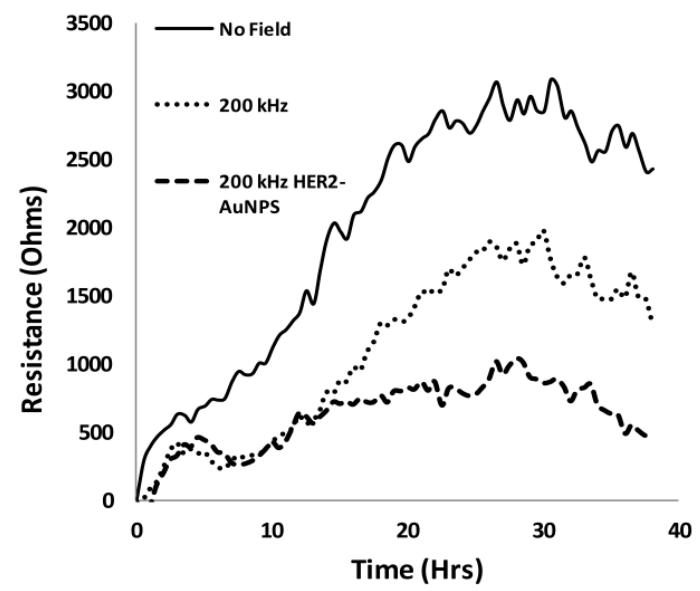

(d)

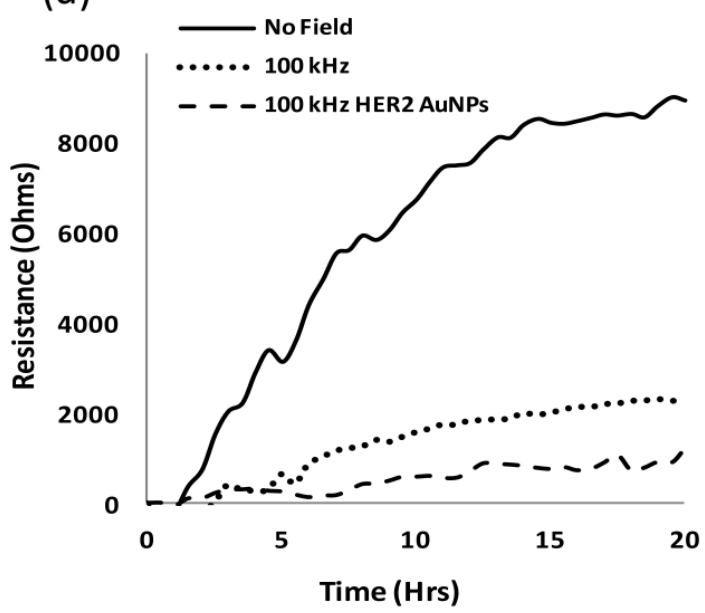

Figure 4. Resistance values for HUVECs (a), SKOV3 (b), MCFIOa (c) and MCF7 (d) under the effects of the optimal frequencies (SKOV3 $200 \mathrm{kHz}$, MCF7 I00 kHz) with and without the application of HER2-AuNPs.

AFM characterization in wet condition was conducted to observe the nanoparticle-cell interaction by quantitative analysis of the roughness of cell membrane upon nanoparticle exposure. Time-lapsed (1, 3 and 24 hours) AFM amplitude images were performed on the cells treated with and without HER2 antibody modified AuNPs. From the amplitude images it is clear that the cells treated with HER2 antibody showed higher number of AuNPs attached on the cell surface when compared to the non-functionalized AuNPs. Compared to the roughness of cell membrane exposed to the normal AuNPs 
(Figure 5b), the higher roughness (Figure 5a) of upon exposure by antibody modified AuNPs indicating the specific binding of functionalized nanoparticles to the cell surface. The HER2 antibody functionalized particles clearly specifically bind to the HER2 receptors on the membrane of cancer cells.

Amplitude images obtained at 24 hours (Figure 5c) showed that upon HER2-AuNPs exposure, there was a significant number of particles present on the cell surface, indicating that the antibody functionalized AuNPs remained on the cell membrane surface during this experiment.

\section{Apoptosis / Necrosis Assay}

Apoptosis and necrosis are the two major processes leading to cell death. Apoptosis is the process of programmed cell death caused by a cascade of biochemical signals within the cell and can be triggered from a variety of external factors usually in the form of stress towards the cell. In the case of apoptosis, the cell prepares itself by performing changes within, one of them being changes in the phospholipid content of the cell membrane.

Apoptotic cells can be easily identified with the protein Annexin V, which is binds to phosphatidylserine, a protein located in the cell membrane. Under normal circumstances, the phosphatidylserine is found in the inner layer of the cell membrane double layer. In the early stages of apoptosis, these phosphatidylserine proteins move to the outer layer of the membrane where Annexin V labeled with fluorescein (FITC) can bind, staining apoptotic cells green.

Necrosis is a traumatic cell death that results from any acute damage caused to the cell where the cell membrane integrity is compromised. This results in the cytosol and organelles within the cell to spill into the surrounding environment. Ethidiumhomodimer III (EthD-III) is a highly positively charged nucleic acid probe which binds to DNA, thus is used to classify necrotic cells by staining them with red fluorescence.

To further understand the effects of the externally applied field to the cells, an Annexin V/ EthD-III assay was performed to determine the cell death mechanism with green fluorescent membrane staining indicating apoptotic cells, and red fluorescent nuclear staining necrotic cells. Figure 6 shows the fluorescent staining of MCF7 cells from one well after 20 hours of exposure to $100 \mathrm{kHz}$ electric field. The image on the left shows EthD-III stained cells (necrotic) and the image on the right shows Annexin V stained cells (apoptotic). There are significantly more cells stained with the Annexin V indicating the death mechanism triggered from the applied electric field to be apoptosis.
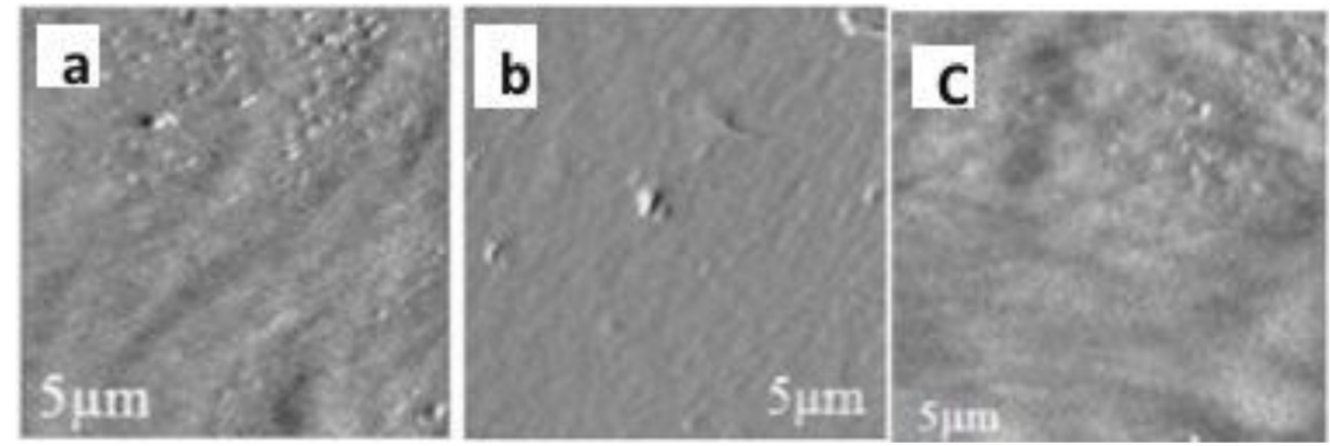

Figure 5. AFM amplitude images showing the surface topography of SKOV-3 cell membrane after treating with and without HER2 modified -AuNPs for 3 hours. It is to be noted that the cells treated with HER2 modified -AuNPs (a) showed more number of particles on the cell surface than the cells treated non modified NPs (b). Figure 5C showing the surface topography of SKOV-3 cell membrane after treating with HER2 modified -AuNPs for 24 hours.
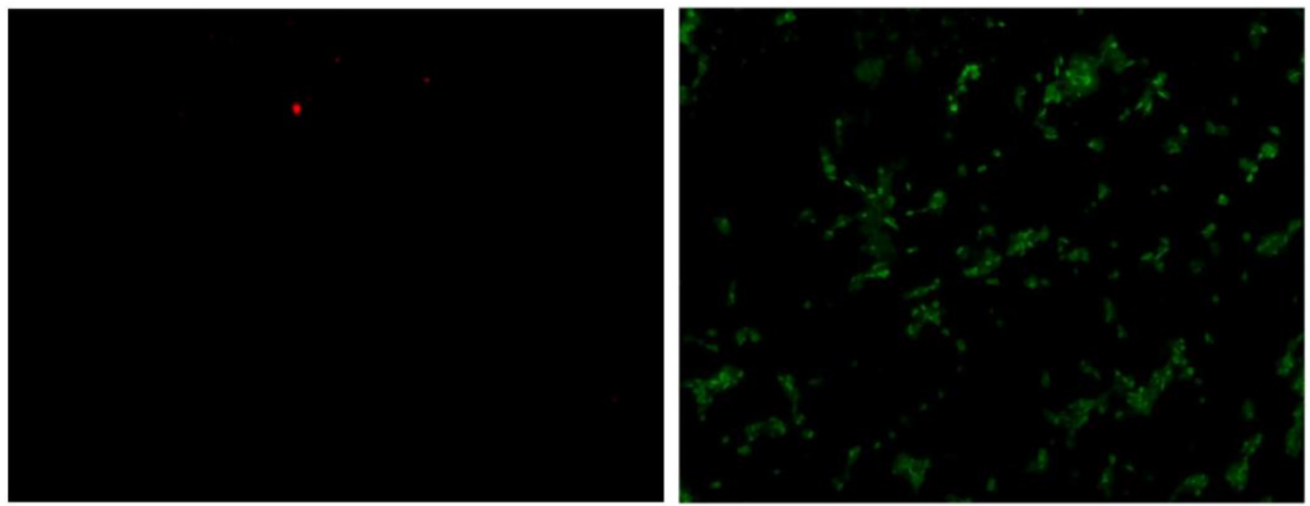

Figure 6. Fluorescent staining of MCF7 cells after 20 hours of exposure to $100 \mathrm{kHz}$ electric field. The image on the left shows EthD-IIl stained cells (necrotic) and the image on the right shows Annexin $V$ stained cells (apoptotic). 


\section{Zeta Potential Measurements}

Due to electrostatic forces, the interior of the cell has uniform potential and a voltage drop across the plasma membrane. With the application of any external electric field, the first electrostatic force encountered would be the membrane potential. Cancerous cells have been shown to have lower cell and cytoplasmic conductivity as well as a lower membrane capacitance compared to non-cancerous cells [37]. This outermost electrically negative zone is composed of negatively charged sialic acid molecules found on the tops of glycoproteins extending outward from the cell membrane. The zeta potential, the potential that exists at the electrical double layer of a particle or cell, is created from these sialic acid residues. Cancer cells have significantly more sialic acid molecules and thus a greater surface negativity. Thus, any external factor that would affect these residues would change the surface negativity of the cell.

In 1990, Seeger and Wolz correlated the electronegativity of cancer cell membranes with membrane degeneration throughout the process of carcinogenesis [38]. This process involves the degradation of the external cell membrane initially, causing more permeability to water-soluble substances to potassium, magnesium, and calcium migrate from the cells and sodium and water accumulate in the cell interior. This is then followed by degenerative changes in the inner membrane of the mitochondria causing loss of anchorage of critical mitochondrial enzymes. Since the membrane potential in a cancer cell is lower than the membrane potential of a healthy cell, the electrical field across the membrane of a cancer cell is reduced. The reduction in membrane electrical field strength will in turn cause alterations in the metabolic functions of the cell.

In order to understand the effects of the applied electric field with targeted nanoparticle enhancement, the next focus of the study was to observe the zeta potential of the cells before and after exposure to the nanoparticles. Zeta potential is the electrostatic potential near the surface of a particle and usually this surface charge is counterbalanced by charges of opposite sign in the surrounding solution. With the nanoparticles, once in contact with the cell membrane, the surface charges of the particles may affect the cells membrane properties with negatively charged ions or molecules will decrease and positively charged ions will increase the surface zeta potential of the cell [39-40], thus attachment of negatively charged nanoparticles on the cell's membrane will cause its zeta potential to become more negative.

We measured the zeta potential of our AuNPs before and after functionalization with the HER2 an- tibody to see if the antibody would affect the nanoparticles' zeta potential. We then measured the zeta potential of the two cancer cell lines, SKOV3 and MCF7, with and without the incorporation of the HER2-AuNPs and compared the results to those for the non cancerous cell lines, HUVEC and MCF10a, respectively (Figure 7). In all cases, the bare AuNPs had the lowest zeta potentials with values of $-37.4 \pm$ $0.7,-40.0 \pm 0.05,-43.3 \pm 0.7$, and $-39.3 \pm 0.4$ as shown in Figure 7 (a), (b), (c) and (d), respectively. With the functionalization of the HER2 antibody, the zeta potentials increased slightly for each case $(-30.5 \pm 1.4$, $-37.1 \pm 1.2,-41.6 \pm 1.0,-35.5 \pm 0.6)$. The cells themselves had initial zeta potentials of $-19.2 \pm 0.9$ (HUVEC), -19.3 \pm 0.8 (SKOV3), - $20.5 \pm 0.9$ (MCF10a) and $-28.3 \pm 1.3$ (MCF7). After incubation with the HER2-AuNPs, the cancerous cell showed a significant decrease in zeta potentials compared to the non-cancerous cells. For the HUVECs, the zeta potential slightly increased to $-18.3 \pm 0.9$, and the MCF10a also showed a slight increase to $-18.9 \pm 2.3$. (Figure 7 (a), (c), respectively). The case was different for both cancerous cell types as the zeta potentials dropped after HER2-AuNPs functionaliztion for the SKOV3 to $-25.3 \pm 1.3$, and for the MCF7 to $-37.6 \pm 1.3$ (Figure $6(\mathrm{~b}),(\mathrm{d})$, respectively).

\section{Discussion}

The cell membrane provides a barrier for the cell and its surroundings and as a result is the first line of defense for the cell to survive. The membrane controls the exchange of electrically charged ions across the cell membrane thus creating the cell membrane potential. It is this potential that is the underlying characteristic of the cell that could distinguish between normal and cancerous cells. It was been noted before that cancerous cell have a lower membrane potential, and it has been shown that the cell's membrane changes during cancer transformation, in particular the membrane's fluidity and charge [41-42]. Therefore, by altering the cell membrane potential, one could possibly control the fate of the cell.

Cancerous cell membranes have a higher number of negatively charged sialic acids, which regulate intercellular contacts and the interaction of charged macromolecules with the cell surface [43]. It is the number of sialic acids that lead to any cell's overall negative membrane potential and ultimately determines a cell's zeta potential [44]. Sialyation is one of the factors that cause the cancer cell membrane to be more electronegative and consequently, any factor that increases or decreases the number of sialic acid residues will change the degree of membrane negativity.

We had proposed that gold nanoparticles functionalized with specific antibodies to target the cancer 
cells would enhance the effects of the electric field towards the cells without affecting the non-cancerous cells due to the different membrane properties of the cancerous cells. In our study, the antibody used targeted HER2 glycoprotein receptor on the cell membrane. Since sialic acids are generally found at the end of most glycoproteins, and the specific cancer cells tested overexpress the HER2 receptor, we can deduce that there would be more HER2-AuNPs attracted to the cancer cells as opposed to the regular cells (Figure 8).

With more negatively charged HER2-AuNPs attached to the cancer cell membrane, the cell membrane could more vulnerable to the affects of the external electric field due to the decrease in surface charge. An important consequence of the existence of electrical charges on the surface of particles is that they will exhibit certain effects under the influence of an applied electric field. Since the changes in zeta potential values depend on cell surface charge, nanoparticle surface charge and their interaction we observed a decrease in zeta potentials of the cells after nanoparticle incorporation leaving the cells more vulnerable to the detrimental effects of the applied electric field.

There was also a noticeable difference in the behavior between the two types of cancer cells. The breast cancer cell line, MCF7 was affected by a lower frequency than the ovarian cancer cell line, SKOV3. This could be due to the MCF7 having a more simpli-

(a)

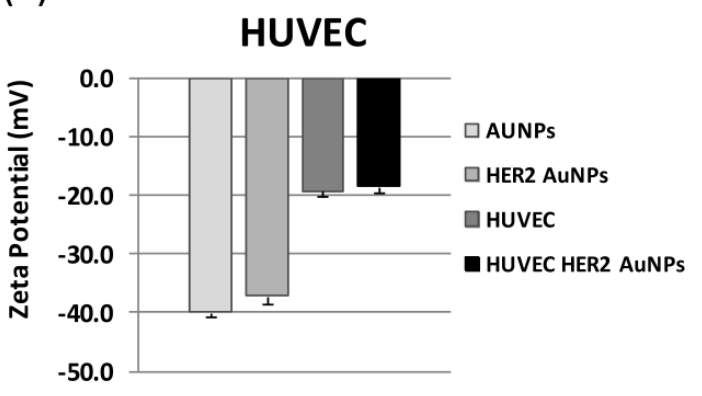

(c)

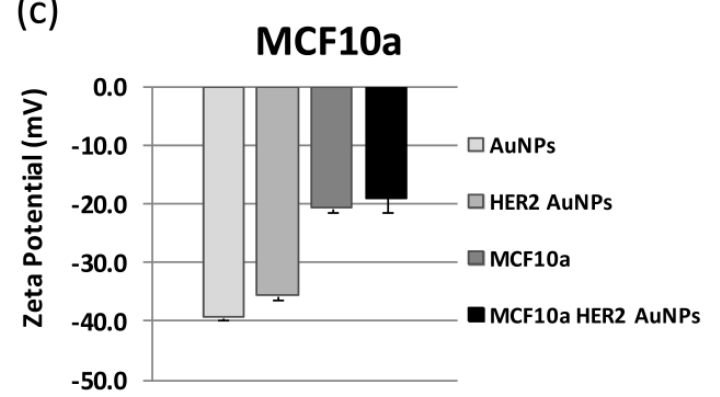

fied cytoskeleton compared with MCF10A, which results in less stiffness and easier deformation [45].

\section{Conclusion}

Cancer electrotherapy treatments are showing considerable promise to avoid the negative side effects of traditional cancer treatments such as chemotherapy. We previously demonstrated that cancer cell proliferation is affected by externally applied alternating electric fields in the intermediate frequency range of $100 \mathrm{kHz}-200 \mathrm{kHz}$. In this study, we demonstrated that different types of cancer cells are affected by different optimal frequencies of these electric fields. To further understand the effects of the applied fields on the cells, we performed an Annexin $\mathrm{V} /$ EthD-III assay to conclude the fate of the cells as apoptosis. We also observed a decrease in proliferation with the addition of HER2-AuNPs to target the cancer cells and enhance the effects of the electric field towards the cells without affecting the non-cancerous cells. With the attached nanoparticles, the zeta potential of the SKOV3 and the MCF7 before and after incorporation of the HER2-AuNPs decreased compared to their non-cancerous counterparts. The decrease in membrane potential would thus leave the cells more vulnerable to the detrimental effects of the applied electric field. The outcome of this research will improve our fundamental understanding of the behavior of cancer cells and define optimal parameters of electrotherapy for clinical and drug delivery applications.

(b)

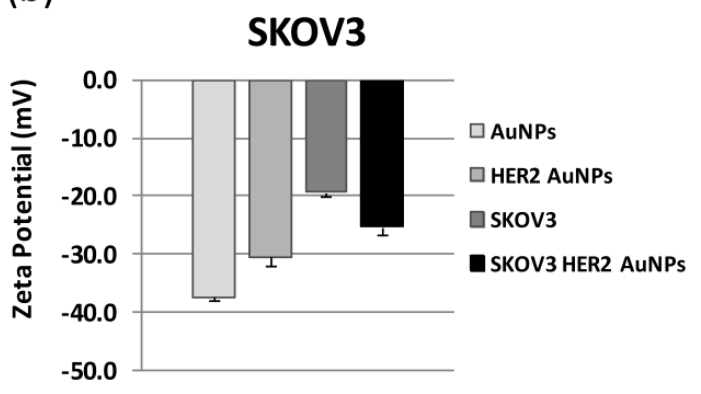

(d)

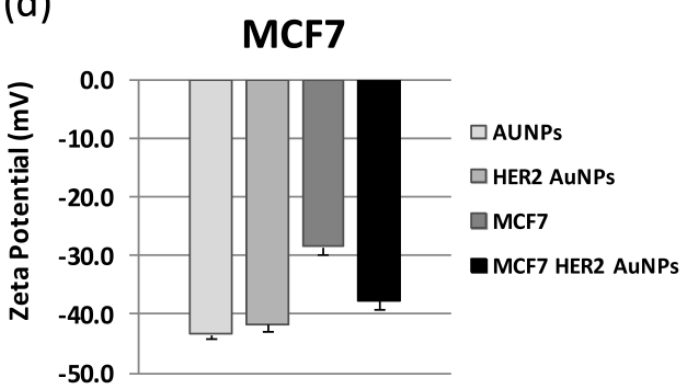

Figure 7. Zeta potential values for HUVECs (a), SKOV3 (b), MCFIOa (c), and MCF7 (d). With the integration of the HER2-AuNPs, the zeta potential of both cancer cells decreased in comparison to the two noncancerous cell types. 


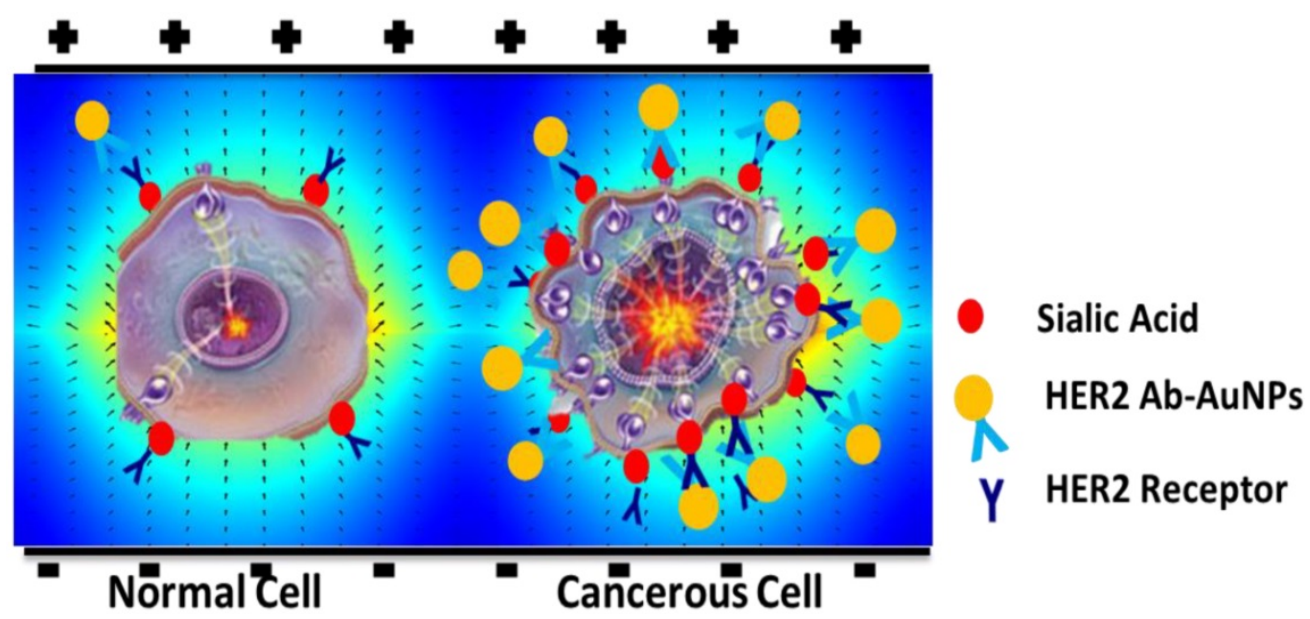

Figure 8. Schematic representation of the cells with and without HER2-AuNPs coating in the field of electrical stimulation.

\section{Acknowledgement}

This study was supported by the grant R15ES021079 from the National Institute of Health (U.S.A.), the grant 1334417 from National Science Foundation (NSF), the grant 21328501 from the National Natural Science Foundation of China and the JSPS Invitation Fellowship Program (L13530) for Research in Japan (Long Term) from the Japan Society for the Promotion of Science (JSPS).

\section{Competing Interests}

The authors have declared that no competing interest exists.

\section{References}

1. Barker AT. Pulsed Magnetic Field Therapy for Tibial Non-Union. Lancet 1984; 8384: 994-996.

2. Ieran M, Zaffuto S, Bagnacani M, Annovi M, Moratti A, Cadossi R. Effect of low frequency pulsing electromagnetic fields on skin ulcers of venous origin in humans: A double-blind study. J Orthop Res. 1990; 8: 276-282

3. Sandyk R. Therapeutic effects of alternating current pulsed electromagnetic fields in multiple sclerosis. J Altern Complement Med. 1997; 3: 365-386.

4. Song L, Chau L, Sakamoto Y, Nakashima J, Koide M, Tuan RS. Electric field-induced molecular vibration for noninvasive, high-efficiency DNA transfection. Mol Ther. 2004; 9: 607-616

5. Golzio M, Rols MP, Teissie J. In vitro and in vivo electric field-mediated permeabilization, gene transfer, and expression. Methods. 2004; 33: 126-135.

6. Teissie J, Escoffre JM, Rols MP, Golzio M. Time dependence of electric field affects on cell membranes. A review for a critical selection of pulse duration for therapeutic applications. Radiol Oncol. 2008; 42: 196-206.

7. Fantozzi F, Arturoni E, Barbucci R. The effects of the electric fields on hydrogels to achieve antitumoral drug release. Bioelectrochemistry. 2010; 78: 191-195.

8. Fricke $\mathrm{H}$, Morse S. The electrical capacity of tumors of the breast. J Cancer Res. 1926; 10: $340-376$.

9. Sundelacruz S, Levin M, Kaplan DL. Role of Membrane Potential in the Regulation of Cell Proliferation and Differentiation. Stem Cell Rev. 2009; 5: 231-246.

10. Cone CDJr, Tongier MJr. Contact inhibition of division: Involvement of the electrical transmembrane potential. J Cell Physio. 1973; 82: 373-386.

11. Qiao G, Duan W, Chatwin C, Sinclair A, Wang W. Electrical properties of breast cancer cells from impedance measurement of cell suspensions. J Phys Conf Ser. 2010; 224: 012081

12. Breton M, Mir LM. Microsecond and Nanosecond Electric Pulses in Cancer Treatments. Bioelectromagnetics. 2012; 33: 106-123

13. Beebe SJ, Fox PM, Rec LJ, Willis EL, Schoenbach KH. Nanosecond, high-intensity pulsed electric fields induce apoptosis in human cells. FASEB J. 2004; 17: 1493-1495.

14. Spadaro M, Ambrosino E, Iezzi M, Di Carlo E, Sacchetti P, Curcio C, Amici A, Wei WZ, Musiani P, Lollini PL, Cavallo F, Forni G. Cure of mammary carcinomas in Her-2 transgenic mice through sequential stimula-tion of innate (neoadjuvant interleukin-12) and adaptive (DNA vaccine electroporation) immunity. Clin Cancer Res. 2005; 11 : 1941-1952.
15. Arcuri M, Cappelletti M, Zampaglione I, Aurisicchio L, Nicosia A, Ciliberto G, Fattori E. Synergistic effect of geneelectro transfer and adjuvant cytokines in increasing the potency of hepatitis C virus genetic vaccination. J Gene Med. 2008; 10: 1048-1054.

16. Sersa G. The state-of-the-art of electrochemotherapy before the ESOPE study; advantages and clinical uses. EJC Suppl. 2006; 4: 52-59.

17. Hondroulis E, Melnick SJ, Wu ZZ, Zhang X, Li CZ. Electrical field manipulation of cancer cell behavior monitored by whole cell biosensing device. Biomed Microdevices. 2013; 15: 657-663.

18. Bianco A, Kostarelos K, Prato M. Applications of carbon nanotubes in drug delivery. Curr Opin Chem Biol. 2005; 9: 674-679.

19. Slowing II, Trewyn BG, Giri S, Lin VSY. Mesoporous Silica Nanoparticles for Drug Delivery and Biosensing Applications. Adv Funct Mater. 2007; 17: 1225-1236.

20. Faraji AH, Wipf P. Nanoparticles in cellular drug delivery. Bioorg Med Chem. 2009; 17: 2950-2962.

21. Harrison BS, Atala A. Carbon nanotube applications for tissue engineering. Biomaterials. 2007; 28: 344-353

22. Kim J, Lee JE, Lee SH, Yu JH, Lee JH, Park TG, Hyeon T. Designed Fabrication of a Multifunctional Polymer Nanomedical Platform for Simultaneous Cancer Targeted Imaging and Magnetically Guided Drug Delivery. Adv Mater. 2008; 20: 478-483.

23. Shi D. Integrated multifunctional nanosystems for medical diagnosis and treatment. Adv Funct Mater. 2009; 19: 3356-3373.

24. De Jong WH, Borm PJA. Drug delivery and nanoparticles: Applications and hazards. Int J Nanomedicine. 2008; 3: 133-149.

25. Hondroulis E, Liu C, Li C.-Z. Whole cell based electrical impedance sensing approach for a rapid nanotoxicity assay. Nanotechnology. 2010;21:315103.

26. Lantz E, Cunningham I, Higa GM. Targeting HER2 in breast cancer: overview of long-term experience. Int J Wom Health. 2010; 1: 155-171.

27. Menard S, Casalini P, Campiglio M, Pupa S, Agresti R, Tagliabue E. HER2 over-expression in various tumor types, focusing on its relationship to the development of invasive breast cancer. Ann Oncol. 2001; 12 (Suppl 1): S15-S19.

28. Tiwari PK, Lee YS. Gene delivery in conjunction with gold nanoparticle and tumor treating electric field. J Appl Phys. 2013; 114: 054902

29. Zhang Y, Yang M, Park JH, Singelyn J, Ma H, Sailor MJ, Ruoslahti E, Ozkan M, Ozkan C. A Surface-Charge Study on Cellular-Uptake Behavior of F3-Peptide-Conjugated Iron Oxide Nanoparticles. Small. 2009; 5: 1990-1996.

30. Zhu X, Hondroulis E, Liu W, Li C.-Z. Biosensing Approaches for Rapid Genotoxicity and Cytotoxicity Assays upon Nanomaterial Exposure. Small, 2013; 9: 1821-1830.

31. Morkvenaitè-Vilkončiené, I, Ramanavičienè, A, Ramanavičius A. Atomic force microscopy as a tool for the investigation of living cells. Medicina (Kaunas). 2013; 49: 155-164.

32. Ghosh PS, Kim CK, Han G, Forbes NS, Rotello VM. Efficient gene delivery vectors by tuning the surface charge density of amino acid-functionalized gold nanoparticles. ACS Nano. 2008; 2: 2213-2218.

33. Kennedy LC, Bickford LR, Lewinski NA, Coughlin AJ, Hu Y, Day ES, West JL, Drezek RA. A New Era for Cancer Treatment: Gold-Nanoparticle-Mediated Thermal Therapies. Small. 2010; 7:2.

34. Hirsch LR, Stafford RJ, Bankson JA, Sershen SR, Rivera B, Price RE, Hazle JD, Halas NJ, West JL. Nanoshell-mediated near-infrared thermal therapy of tumors under magnetic resonance guidance. Proc Natl Acad Sci U S A. 2003; 100: 13549.

35. Gannon CJ, Patra CR, Bhattacharya R, Mukherjee P, Curley SA. Intracellular gold nanoparticles enhance non-invasive radiofrequency thermal destruction of human gastrointestinal98 cancer cells. J Nanobiotechnology. 2008; 6: 2.

36. Jolesz FA, Hynynen K. Magnetic resonance image-guided focused ultra-sound surgery. Cancer J. 2002; 8 (Suppl 1): S100

37. Wonderlin WF, Woodfork KA, Strobl JS. Changes in membrane potential during the progression of MCF-7 human mammary tumor cell through the cell cycle. J Cell Physiol. 1995; 165: 177-185.

38. Seeger PG, Wolz S. Successful Biological Control of Cancer: By Combat against the Causes. Gesamtherstellung: Neuwieder Verlagsgesellschaft mbH. 1990

39. Altankov G, Richau K, Groth T. The role of surface zeta potential and substratum chemistry for regulation of dermal fibroblasts interaction. Mater Werks. 2003; 34: $1120-1128$. 
40. SilivaFilho FC, Santos AB, de Carvalho TM, de Souza W. Surface charge of resident, elicited, and activated mouse peritoneal macrophages. J Leukocyte Biol. 1987; 41: $143-149$.

41. Dobrzynska I, Szachowicz-Petelska B, Figaszewski Z, Sulkowski S. Changes in electric charge and phospholipid composition in human colorectal cancer cells. Mol Cell Biochem. 2005; 276: 113-119.

42. Szachowicz-Petelska B, Figaszewski Z, Sulkowski S. Altered membrane free unsaturated fatty acid composition in human colorectal cancer tissue. Mol Cell Biochem. 2007; 294: 237-242.

43. Rosenberg SA, Einstein AB Jr. Sialic Acids on the Plasma Membrane of Cultured Human Lymphoid Cells: Chemical Aspects and Biosynthesis. J Cell Biol. 1972; 53: 466-473.

44. Cure JC. On the electrical characteristics of cancer. Second International Congress of Electrochemical Treatment of Cancer, Jupiter, Florida. 1995

45. Qiao G, Wang W, Duan W, Zheng F, Sinclair AJ, Chatwin CR. Bioimpedance Analysis for the Characterization of Breast Cancer Cells in Suspension. IEEE Trans BioMed Eng. 2012; 59: 2321-2329.

46. Zhu X, Hondroulis E, Li C.-Z. Biosensing Approach for Rapid Genotoxicity and Cytotoxicity Assay upon Nanomaterial Exposure. Small, 2013, 9: 1821-1830.

47. Hondroulis E, Li C.-Z. Impedance based Nanotoxicity Assessment of Graphene Nanomaterials at Cellular and Tissue Level. Analytical Letters, 2011, 45: 272-282.

48. Hondroulis E, Liu C, Li C.-Z. Whole Cell Based Electrical Impedance Sensing Approach for Rapid Nanotoxicity Assay. Nanotechnology, 2010, 31:315103. 